\title{
An experimental and computational study of the effect of aqueous solution on the multiphoton ionisation photoelectron spectrum of phenol $\uparrow$
}

\author{
Alice Henley, (D) † Jamie W. Riley, (D) + Bingxing Wang (D) $\S$ \\ and Helen $\mathrm{H}$. Fielding (iD *
}

Received 10th June 2019, Accepted 15th July 2019

DOI: $10.1039 /$ c9fd00079h

\begin{abstract}
We revisit the photoelectron spectroscopy of aqueous phenol in an effort to improve our understanding of the impact of inhomogeneous broadening and inelastic scattering on solution-phase photoelectron spectra. Following resonance-enhanced multiphoton ionisation via the $1^{1} \pi \pi^{*}$ and $1^{1} \pi \sigma^{*}$ states of phenol, we observe $1^{1} \pi \pi^{*}-D_{0} / D_{1}$ ionisation and competing direct $S_{0}-D_{0} / D_{1}$ ionisation. Following resonance-enhanced multiphoton ionisation via the $2^{1} \pi \pi^{*}$ state, we observe the signature of solvated electrons. By comparing the photoelectron spectra of aqueous phenol with those of gas-phase phenol, we find that inelastic scattering results in peak shifts with similar values to those that have been observed in photoelectron spectra of solvated electrons, highlighting the need for a robust way of deconvoluting the effect of inelastic scattering from liquid-phase photoelectron spectra. We also present a computational strategy for calculating vertical ionisation energies using a quantum-mechanics/effective fragmentation potential (QM/EFP) approach, in which we find that optimising the configurations obtained from molecular dynamics simulations and using the [phenol $\left.\cdot\left(\mathrm{H}_{2} \mathrm{O}\right)_{5}\right]_{Q M}\left[\left(\mathrm{H}_{2} \mathrm{O}\right)_{n \geq 250}\right]_{\text {EFP }}(\mathrm{B} 3 \mathrm{LYP} / \mathrm{aug}-\mathrm{cC}-\mathrm{pvdz})$ method gives good agreement with experiment.
\end{abstract}

Department of Chemistry, University College London, 20 Gordon Street, London WC1H OAJ, UK. E-mail: h.h. fielding@ucl.ac.uk

$\dagger$ Electronic supplementary information (ESI) available: Streaming potential measurement; photoelectron spectra plotted as a function of eKE; details of fitting procedures; convergence of VIE; tables of calculated and experimental VIEs and VEEs; selection of probable configurations; consistencies between software packages; molecular orbitals; coordinates of QM region. See DOI: $10.1039 / \mathrm{c} 9 \mathrm{fd} 00079 \mathrm{~h}$

$\$$ These authors contributed equally to this work.

$\S$ Present address: College of Chemistry and Chemical Engineering, Henan Institute of Science and Technology, Eastern Hualan Avenue, Xinxiang, 453003, China. 
Ionisation is the most fundamental photophysical process accompanying the interaction of ionising radiation with biologically important molecules and plays a central role in radiation chemistry and biology. The formation of an electron and a radical is the first step in a chain of chemical reactions that results in DNA damage. The most direct way to probe ionisation experimentally is to use photoelectron spectroscopy (PES), which measures the electron kinetic energies (eKEs) of electrons emitted following ionisation. The eKE distribution encodes the role of each vibrational mode of the radical in its subsequent structural relaxation and, in the case of solution-phase photoelectron spectra, also contains information about solvent relaxation. However, the interpretation of solutionphase photoelectron spectra is complicated by the inhomogeneous environment of the solution causing spectral broadening and inelastic scattering of photoelectrons in the solution before emission causing the measured electron kinetic energies to be lower than their true values. ${ }^{1,2}$ Disentangling the various contributions to solution-phase photoelectron spectra requires gas-phase PES as an essential reference and synergistic contributions from experiment and theory. Here, we revisit the PES of aqueous phenol in an effort to improve our understanding of the impact of inhomogeneous broadening and inelastic scattering on the photoelectron spectra.

Phenol is a ubiquitous molecular motif in many biologically relevant chromophores. It is the chromophore in the amino acid tyrosine, which plays an important role in photosynthesis, ${ }^{3}$ and it is a building block of the chromophore in green fluorescent protein, the most widely used fluorescent probe for in vivo monitoring of biological and biochemical processes. ${ }^{4,5}$ The UV absorption spectrum of phenol is dominated by two bands centered around $270 \mathrm{~nm}(4.6 \mathrm{eV})$ and $210 \mathrm{~nm}(5.9 \mathrm{eV})$, corresponding to transitions from the electronic ground state, $\mathrm{S}_{0}$, to the first two ${ }^{1} \pi \pi^{*}$ states, labelled $1^{1} \pi \pi^{*}$ and $2^{1} \pi \pi^{*}$ (Fig. 1). Between these two ${ }^{1} \pi \pi^{*}$ states lies a ${ }^{1} \pi \sigma^{*}$ state, labelled $1^{1} \pi \sigma^{*}$. The $1^{1} \pi \sigma^{*}$ state is composed of $\mathrm{O}-$ centered $\pi$ 3s and $\pi \sigma^{*}$ configurations and is dissociative along the $\mathrm{O}-\mathrm{H}$ stretch coordinate. The $1^{1} \pi \sigma^{*}$ state forms conical intersections (CIs) with the $1^{1} \pi \pi^{*}$ and $\mathrm{S}_{0}$ states at modest $\mathrm{O}-\mathrm{H}$ bond lengths and, therefore, plays an important role in the photostability of chromophores containing the phenol motif. ${ }^{6,7}$

There have been numerous experimental and computational studies of the photochemistry and photophysics of isolated phenol molecules in vacuo and in solution. ${ }^{6,8-34}$ Gas-phase studies have revealed that following photoexcitation above the $1^{1} \pi \pi^{*} / 1^{1} \pi \sigma^{*} \mathrm{CI}$, the dissociative $1^{1} \pi \sigma^{*}$ potential energy surface is accessed directly and $\mathrm{O}-\mathrm{H}$ bond fission occurs, forming phenoxyl radical and hydrogen atom products $\left(\mathrm{PhO}^{*}+\mathrm{H}\right)$ on a femtosecond timescale. ${ }^{20}$ Following photoexcitation just below the $1^{1} \pi \pi^{*} / 1^{1} \pi \sigma^{*} \mathrm{CI}$, the dissociative $1^{1} \pi \sigma^{*}$ potential energy surface is accessed by tunnelling through the barrier under the CI, forming $\mathrm{PhO}^{\circ}+\mathrm{H}$ on a nanosecond timescale. ${ }^{14,22,23}$ In hexane, an aprotic solvent, the initial bond fission processes and timescales have been found to be very similar to those in the gas phase. ${ }^{35}$ However, in aqueous solution, new relaxation pathways are possible. Following photoexcitation of the $2^{1} \pi \pi^{*}$ state at $200 \mathrm{~nm}$ and the $1^{1} \pi \pi^{*}$ state below the $1^{1} \pi \pi^{*} / 1^{1} \pi \sigma^{*} \mathrm{CI}$, solvated electrons and $\mathrm{PhO}^{*}$ radicals were observed to be formed on timescales of $200 \mathrm{fs}$ and $2 \mathrm{~ns}$, respectively, using 


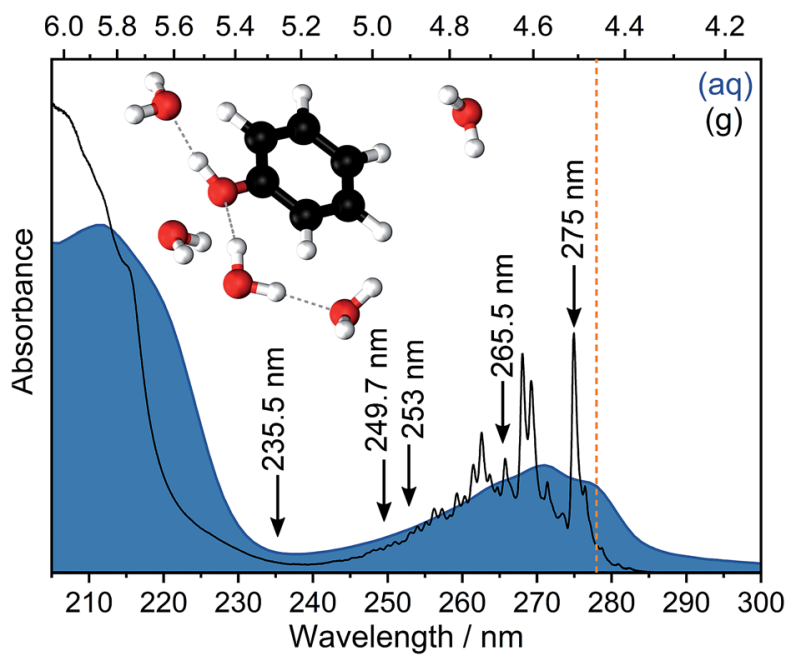

Fig. 1 UV absorption spectra of phenol in the gas phase (g) and in aqueous solution (aq). Arrows indicate the wavelengths employed in our multiphoton PES measurements and the dashed vertical line indicates our estimate of the $S_{0}-S_{1}$ adiabatic excitation energy (AEE). Inset: geometry of phenol $\left(\mathrm{H}_{2} \mathrm{O}\right)_{5}$ optimised at the [phenol $\left.\cdot\left(\mathrm{H}_{2} \mathrm{O}\right)_{5}\right]_{Q M}\left[\left(\mathrm{H}_{2} \mathrm{O}\right)_{263}\right]_{\mathrm{EFP}}$ level of theory.

transient absorption spectroscopy. ${ }^{28}$ In both cases, autoionisation was proposed as a mechanism for the formation of solvated electrons. A recent liquid-microjet PES study by our group found that following photoexcitation of the $1^{1} \pi \sigma^{*}$ state at $235 \mathrm{~nm}$, just above the $1^{1} \pi \pi^{*} / 1^{1} \pi \sigma^{*} \mathrm{CI}$, IC to the $1^{1} \pi \pi^{*}$ state occurred on a $150 \mathrm{fs}$ timescale. ${ }^{31}$ It was also suggested that solvated electrons were formed on the same ultrafast timescale by a sequential mechanism, involving $\mathrm{O}-\mathrm{H}$ bond fission to form $\mathrm{PhO}^{*}+\mathrm{H}$ followed by proton-coupled electron transfer (PCET).

Although the electronic relaxation dynamics of photoexcited neutral phenol molecules has been studied extensively, there has been less interest in the photoionisation of phenol. PES ${ }^{36-40}$ and multiphoton ionisation (MPI) mass-spectrometry ${ }^{41}$ measurements of gas-phase phenol have determined the first two adiabatic ionisation energies (AIEs) to be $8.508 \mathrm{eV}$ (ref. 41) and $9.36 \mathrm{eV},{ }^{36}$ for the ground and first electronically excited states of the radical ion, $\mathrm{D}_{0}$ and $\mathrm{D}_{1}$, respectively. A resonance-enhanced MPI (REMPI) PES study of gas-phase phenol revealed the vertical ionisation energy (VIE) from the $1^{1} \pi \pi^{*}$ state to $D_{0}$ to be around $0.3 \mathrm{eV}$ higher than the AIE. ${ }^{42}$ Recent quantum dynamics calculations have identified the key vibrational modes contributing to the subsequent electronic relaxation of the radical ion following photoionisation..$^{33}$ In aqueous solution, an $\mathrm{X}$-ray PES study of aqueous phenol using a liquid-microjet revealed the VIEs for $\mathrm{D}_{0}$ and $\mathrm{D}_{1}$ to be lowered to $7.8 \pm 0.1 \mathrm{eV}$ and $8.6 \pm 0.1 \mathrm{eV}$, respectively. ${ }^{43}$ Two independent liquid-microjet MPI PES studies of phenol in aqueous solution gave VIEs of $7.6 \pm 0.1 \mathrm{eV}, 8.5 \pm 0.1 \mathrm{eV}$ (ref. 31 ) and $8.0 \pm 0.1 \mathrm{eV}, 8.5 \pm 0.1 \mathrm{eV} .{ }^{44}$ Despite both studies yielding VIEs within experimental error of the X-ray PES data, the two experiments did not yield values that were in good agreement with each other. The photoelectron spectra recorded by us were analysed by fitting the data to 
a single photoionisation process from $1^{1} \pi \pi^{*}$, to $\mathrm{D}_{0} \cdot{ }^{31}$ The photoelectron spectra recorded by Roy et al. were analysed by fitting the data to two ionisation processes from $1^{1} \pi \pi^{*}$, to $\mathrm{D}_{0}$ and $\mathrm{D}_{1}$, and by including a shift to account for inelastic scattering, estimated from photoelectron spectra of solvated electrons in aqueous solution. ${ }^{44}$ Calculations of phenol $\cdot\left(\mathrm{H}_{2} \mathrm{O}\right)_{4}$ clusters, in which the VIEs were determined using the equation-of-motion coupled-cluster method with single and double excitations for ionisation potentials (EOM-IP-CCSD) ${ }^{45}$ method for phenol perturbed by the electrostatic field of a $35 \AA$ spherical box of water molecules modelled using the effective fragment potential (EFP) method, have given VIEs to $\mathrm{D}_{0}$ and $\mathrm{D}_{1}$ of $7.9 \mathrm{eV}$ and $8.6 \mathrm{eV}$, respectively, ${ }^{43}$ in agreement with the experimental measurements.

The different approaches to the analysis of liquid-microjet MPI photoelectron spectra $^{31,44}$ motivated us to revisit the MPI PES of aqueous phenol. In this paper, we compare the results of new liquid-microjet MPI PES experiments with gasphase MPI PES measurements ${ }^{31}$ and liquid-microjet X-ray PES measurements. ${ }^{43}$ We also compare photoionisation calculations of phenol in the gas phase with those in aqueous solution using density functional theory (DFT) and EOM-IPCCSD methods for phenol $\cdot\left(\mathrm{H}_{2} \mathrm{O}\right)_{5}$ clusters perturbed by the electrostatic field of water molecules modelled using the EFP method.

\section{Methods}

\subsection{Experimental}

Photoelectron spectra were recorded using our recirculating liquid-microjet magnetic-bottle time-of-flight (TOF) photoelectron spectrometer that has been described in detail elsewhere. ${ }^{46}$ Briefly, a $100 \mathrm{mM}$ aqueous phenol solution, with $30 \mathrm{mM}$ sodium fluoride added to minimise charging effects and increase the conductivity, was introduced through a $20 \mu \mathrm{m}$ diameter quartz nozzle into the spectrometer. The liquid-microjet was intersected with femtosecond laser pulses approximately $1 \mathrm{~mm}$ below the nozzle, in the region of laminar flow. The femtosecond laser pulses were generated by frequency upconverting the output of an optical parametric amplifier pumped by an amplified Ti:sapphire femtosecond laser system operating at $1 \mathrm{kHz}$; the electric field vectors of the laser pulses were parallel to the TOF axis and the $1 / e^{2}$ pulse duration of the $235.5 \mathrm{~nm}$ pulses was measured to be $\sim 150$ fs. Photoelectrons were detected at the end of the TOF tube and the photoelectron current was recorded together with the arrival time relative to the trigger of the laser pulse. The photoelectron count-rate was kept at around $500 \mathrm{~Hz}$ to avoid space-charge effects and saturation of the detector. eKE spectra were determined by calibrating the TOF against the MPI photoelectron spectrum of $\mathrm{NO}^{47}$ and multiplying the photoelectron counts by the Jacobian $m_{\mathrm{e}} s^{2} /\left(t-t_{0}\right)^{3}$, where $m_{\mathrm{e}}$ is the mass of an electron, $t$ is the TOF and $s$ and $t_{0}$ are calibration constants. Photoelectron spectra of Xe were recorded to determine the energy resolution and streaming potential, which were $\Delta E / E \sim 1 \%$ and $\phi_{\text {str }}=0, \dagger$ respectively.

Compared with our previous work, ${ }^{31}$ we have employed a recirculating system instead of a liquid nitrogen cold-trap, which improves the quality of the photoelectron spectra at low eKE. We have also rewritten the data analysis software and corrected an error in the way the Jacobian was implemented, which results in higher photoelectron counts at low eKE (see ref. 46). 


\subsection{Computational}

To benchmark the quantum mechanical (QM) computational methods used to calculate the VIEs and vertical excitation energies (VEEs) of aqueous phenol, calculations of isolated phenol molecules in the gas phase were performed at the same levels of theory. The structure of gas-phase phenol was optimised using the $\mathrm{B}^{2} \mathrm{LYP}^{48-51} / 6-311++\mathrm{G}(3 \mathrm{df}, 3 \mathrm{pd})^{52-54}$ method and frequency-calculations were performed to ensure that a minimum on the potential energy surface was reached. The VIE was determined using two methods: B3LYP/aug-cc-pvdz to determine the energy difference between neutral phenol and its corresponding cation, at the minimum energy geometry of neutral phenol, and the EOM-IP-CCSD $/ 6-31+\mathrm{G}^{*}$ method. VEEs were calculated using the equation-of-motion coupled-cluster method with single and double excitations for excitation energies (EOM-EECCSD $)^{45}$ and the algebraic diagrammatic construction method to second order $(A D C(2)),{ }^{55,56}$ both with the $6-31+G^{*}$ basis set. All gas-phase calculations were performed using the QChem software package ${ }^{57}$ apart from the optimisation and frequency calculations which were performed using Gaussian 09.58

Several steps were involved in the calculation of VIEs and VEEs of phenol in aqueous solution. First, a classical molecular dynamics (MD) simulation (NAMD, ${ }^{59}$ developed by the Theoretical and Computational Biophysics Group in the Beckman Institute for Advanced Science and Technology at the University of Illinois at Urbana-Champaign) was used to sample an ensemble of conformations of phenol in bulk water (Fig. 2). In the MD simulation, phenol was soaked in a sphere of water with radius $50 \AA$ (17 877 water molecules) and the CHARMM force field was used to model the system. ${ }^{60-62}$ The system was minimised for 2 ps and then allowed to equilibrate at $300 \mathrm{~K}$ for a further $20 \mathrm{ps}$ before running a trajectory for 150 ps. Frames from the trajectory were saved every 500 fs (300 frames in total) for subsequent quantum mechanical/EFP (QM/EFP) calculations. Hybrid QM/EFP methods provide a rigorous yet computationally affordable way to include solute-solvent interactions. ${ }^{63}$ In the EFP region, solvent molecules are modelled as discrete entities using non-empirical model potentials that perturb the QM region by their electrostatic potentials. The EFP also includes polarisation, dispersion and exchange interaction energies at the QM/EFP interface and between the individual EFP fragments.

For the QM/EFP calculations, the QM region was selected to be phenol plus the five water molecules closest to any atom within phenol. Although fewer water molecules have been employed for other calculations of phenol in aqueous solution, ${ }^{34,43}$ we used five because we found this was enough to ensure that all the water molecules that are hydrogen-bonded to phenol (donor-acceptor distance < $3 \AA$, donor-H-acceptor bond angle $180 \pm 20^{\circ}$ ) were included in the QM region. The EFP region was selected to be all other water molecules within $10 \AA$ of any atom in the phenol molecule (250-300 water molecules). A radius of $10 \AA$ was selected because it has been shown to work well for similar calculations for the green fluorescent protein chromophore in bulk water. ${ }^{64}$ The water molecules outside the EFP region were then discarded. The EFP parameters used to represent water were the standard parameters in the Q-Chem ${ }^{65}$ or Firefly ${ }^{66}$ libraries.

The QM and EFP selections were made independently for each of the 300 frames saved from the MD simulation and therefore each frame has a different phenol conformation as well as different configurations of water molecules, in both the QM 


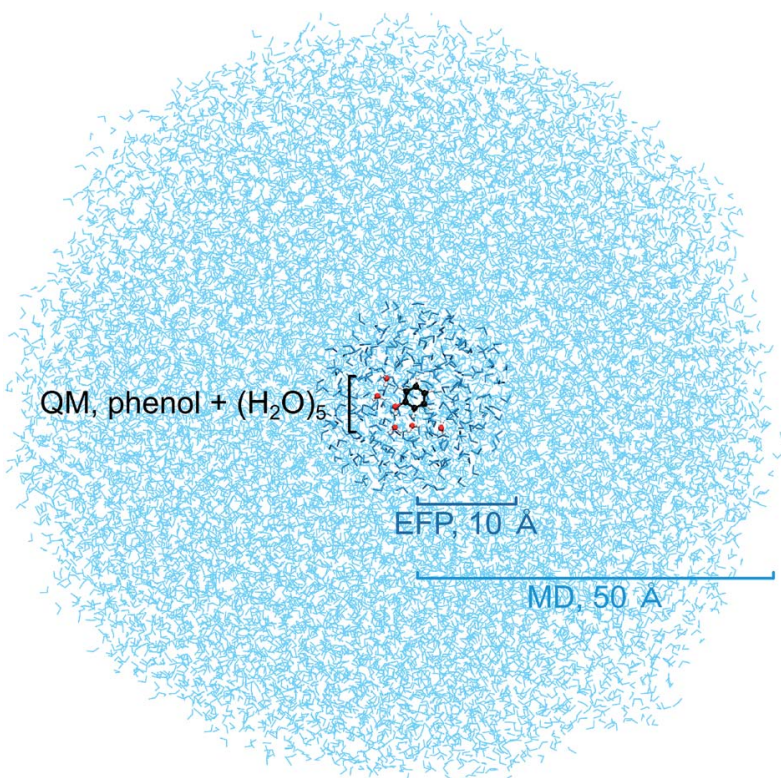

Fig. 2 Simulation box showing the QM region containing a cluster of phenol and 5 water molecules, a sphere of radius $10 \AA$ containing 250-300 water molecules modelled using the EFP method and a sphere of radius $50 \AA$ containing the water molecules used in the initial MD simulations.

and EFP regions. The energies of each of the 300 configurations were calculated using QM/EFP at the B3LYP/aug-cc-pvdz level of theory for both $\mathrm{S}_{0}$ (Fig. S7 $\dagger$ ) and $\mathrm{D}_{0}$ using the QChem software package. VIEs were then calculated as the difference between these $S_{0}$ and $D_{0}$ energies. Higher level calculations were then performed using a selection of the 300 frames. To make these calculations computationally affordable, we selected a relatively small set of frames and a smaller basis set. The ten configurations with $S_{0}$ energies closest to the mean $S_{0}$ energy at the QM/EFP (B3LYP/aug-cc-pvdz) level were selected (Fig. S7 $\dagger$ ) for QM/EFP calculations using EOM-IP-CCSD, EOM-EE-CCSD and ADC(2), with the $6-31+\mathrm{G}^{*}$ basis set. These calculations were performed using the QChem software package.

To investigate whether optimisation with QM/EFP improved the calculations, additional QM/EFP calculations of VIEs and VEEs, using B3LYP/aug-cc-pvdz, EOM-IP-CCSD $/ 6-31+G^{*}$, EOM-EE-CCSD $/ 6-31+\mathrm{G}^{*}$ and $\mathrm{ADC}(2) / 6-31+\mathrm{G}^{*}$, were performed for the same ten frames following optimisation at the PBE0 (ref. 67-69)/ aug-cc-pvdz level of theory. The geometry optimisation was carried out using the Firefly quantum chemistry package, ${ }^{70}$ which is partially based on the GAMESS (US) source code ${ }^{71}$ it adjusts the positions of all atoms within the QM region at the PBE0/aug-cc-pvdz level and the 250-300 explicit water molecules modelled by EFP are also reorientated (rotational and translational degrees of freedom).

\section{Results and discussion}

In Fig. 3, we present $1+1$ MPI photoelectron spectra of phenol in aqueous solution as a function of one-photon electron binding energy, eBE $=h \nu-\mathrm{eKE}$ 

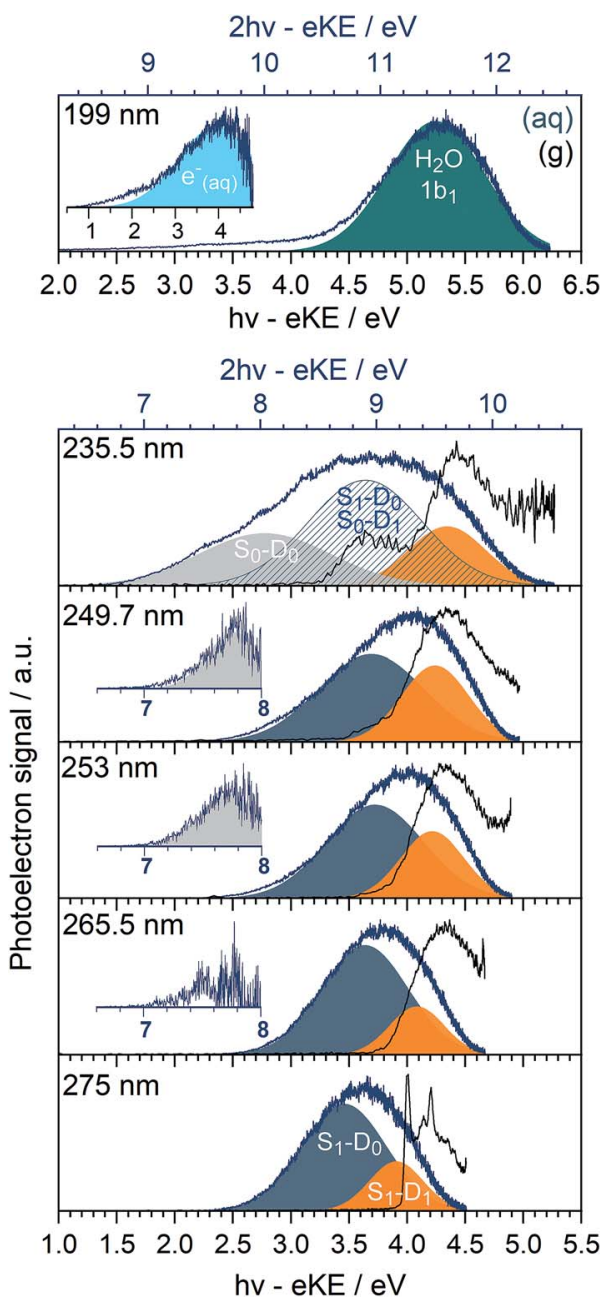

Fig. $3 \mathrm{MPI}$ photoelectron spectra of phenol in the gas phase (black) and in aqueous solution (blue) recorded following photoexcitation at $275 \mathrm{~nm}(4.51 \mathrm{eV}), 265.5 \mathrm{~nm}(4.67 \mathrm{eV})$, $253 \mathrm{~nm}$ (4.90 eV), $249.7 \mathrm{~nm}$ (4.97 eV), $235.5 \mathrm{~nm}$ (5.26 eV) and $199 \mathrm{~nm}$ (6.23 eV), plotted as a function of one-photon eBE (lower axes). The additional scales marked on the top horizontal axes of the $235.5 \mathrm{~nm}$ and $199 \mathrm{~nm}$ spectra (blue) represent the two-photon eBEs for these photon energies. Intensities of the individual spectra have been normalised to their maxima. Gaussians represent $\mathrm{S}_{1}\left(1^{1} \pi \pi^{*}\right)-\mathrm{D}_{0}$ (dark blue), $\mathrm{S}_{1}\left(1^{1} \pi \pi^{*}\right)-\mathrm{D}_{1}$ (orange), $\mathrm{S}_{0}-\mathrm{D}_{0}$ (light grey). Insets in 265.5-249.7 nm spectra are residuals of the fits of Gaussians to the low eBE edges of the photoelectron spectra, corresponding to $S_{0}-D_{0}$. The $S_{0}-D_{1}$ ionisation process overlaps with the $S_{1}\left(1^{1} \pi \pi^{*}\right)-D_{0}$ process in the $235.5 \mathrm{~nm}$ PES (stripes). The dark green Gaussian in the $199 \mathrm{~nm}$ photoelectron spectrum corresponds to two-photon ionisation from the $1 b_{1}$ molecular orbital of $\mathrm{H}_{2} \mathrm{O}(\mathrm{l})$ and the inset corresponds to the residual of the low eBE edge (plotted as a function of one-photon eBE) and is attributed to $\mathrm{e}^{-}(\mathrm{aq}) \rightarrow \mathrm{e}^{-}(\mathrm{g})$

where $h \nu$ is photon energy, together with the equivalent gas-phase photoelectron spectra. ${ }^{31}$ The photoelectron spectra of phenol in aqueous solution are shifted to lower eBEs by around $0.8 \mathrm{eV}$ compared to the gas phase and are similar to those 
reported in our earlier work; ${ }^{31}$ however, as a result of the improved quality of data obtained using a recirculator compared to a liquid nitrogen cold-trap (Section 2.1) we are able to identify additional features. The $275-249.7 \mathrm{~nm}$ photoelectron spectra recorded following resonance-enhanced MPI via the $1^{1} \pi \pi^{*}$ state are now best fit with two Gaussians, corresponding to $1^{1} \pi \pi^{*}-\mathrm{D}_{0} / \mathrm{D}_{1}$ ionisation processes. At $275 \mathrm{~nm}$, the area of the peak corresponding to ionisation to $\mathrm{D}_{0}$ is around three times larger than that of the peak corresponding to ionisation to $\mathrm{D}_{1}$, in agreement with our earlier calculations of photoionisation cross-sections from the $1^{1} \pi \pi *$ state. ${ }^{31}$ This contrasts with the $267 \mathrm{~nm}$ MPI photoelectron spectrum reported by Roy et al. in which the area of the peak corresponding to ionisation to $\mathrm{D}_{0}$ was substantially less than the area of the peak corresponding to ionisation to $\mathrm{D}_{1}{ }^{\mathbf{4 4}}$ The ratios of the areas of the two peaks corresponding to ionisation from $1^{1} \pi \pi^{*}$ to $D_{0}$ and $D_{1}$ are observed to decrease with increasing photon energy (Fig. 3), unlike the calculations; ${ }^{31}$ this could be attributed to increased solute and solvent reorganisation during ionisation to $D_{1}$ compared to ionisation to $D_{0}$. The residuals of the fits at the low eBE edges of the 265.5-249.7 $\mathrm{nm}$ spectra are plotted as insets in Fig. 3 and can be attributed to non-resonant $S_{0}-D_{0}$ MPI that competes with resonance-enhanced MPI. The contribution from non-resonant MPI increases with decreasing $\mathrm{S}_{0}-1^{1} \pi \pi^{*}$ absorption cross-section, as we would expect. This feature was not observed in either of the previous MPI studies of aqueous phenol but is observed in the MPI gas-phase PES. ${ }^{31,44}$

The $235.5 \mathrm{~nm}(5.26 \mathrm{eV})$ MPI photoelectron spectrum is very broad and can be fit with either three or four Gaussians; however, we believe that fitting to three Gaussians is more appropriate (see below). There are four processes contributing to the photoelectron spectrum fit to three Gaussians: resonant $1^{1} \pi \pi^{*}-\mathrm{D}_{0} / \mathrm{D}_{1}$ MPI and non-resonant $S_{0}-D_{0} / D_{1}$ MPI. At this photon energy, the photoelectron spectra corresponding to $1^{1} \pi \pi^{*}-\mathrm{D}_{0}$ and $\mathrm{S}_{0}-\mathrm{D}_{1}$ lie on top of one another and it is not possible to distinguish between them by fitting an additional Gaussian. The peak centered at $8.0 \pm 0.1 \mathrm{eV}$ two-photon eBE corresponds to $\mathrm{S}_{0}-\mathrm{D}_{0}$ ionisation and is close to the X-ray PES measurement. ${ }^{43}$ At $235.5 \mathrm{~nm}$, the $1^{1} \pi \pi^{*}$ state is not populated directly (Fig. 1) but our observation of $1^{1} \pi \pi^{*}-\mathrm{D}_{0} / \mathrm{D}_{1}$ ionisation is consistent with photoexcitation of the $1^{1} \pi \sigma^{*}$ state followed by rapid relaxation to the $1^{1} \pi \pi^{*} / 1^{1} \pi \sigma^{*} \mathrm{CI}$, after which some population will undergo IC to the $1^{1} \pi \pi^{*}$ state before photoionisation, on the timescale of the measurement $(\sim 150 \mathrm{fs})$.

In our earlier work, the $235.5 \mathrm{~nm}$ MPI photoelectron spectrum was fit to four Gaussians. The additional Gaussian was attributed to the photoelectron spectrum of the solvated electron that we proposed was formed following relaxation through the $1^{1} \pi \pi^{*} / 1^{1} \pi \sigma^{*} \mathrm{CI}$ after which, in addition to IC to the $1^{1} \pi \pi^{*}$ state, $\mathrm{O}-\mathrm{H}$ dissociation could occur to form $\mathrm{PhO}^{*}+\mathrm{H}$, followed by proton-coupled electron transfer, $\mathrm{H}(\mathrm{aq})+\mathrm{H}_{2} \mathrm{O} \rightarrow \mathrm{H}_{2} \mathrm{O}^{+}(\mathrm{aq})+\mathrm{e}^{-}(\mathrm{aq})$. It is possible that there is a contribution from solvated electrons to the peak we have assigned as $\mathrm{S}_{1}-\mathrm{D}_{1}$.

The $199 \mathrm{~nm}(6.23 \mathrm{eV})$ MPI photoelectron spectrum, which has not been reported before, is dominated by a peak centred around $5.25 \pm 0.1 \mathrm{eV}$ eBE with a long tail at low eBE. The peak centred around $5.25 \pm 0.1 \mathrm{eV}$ eBE can be fit with a single Gaussian and corresponds to $11.5 \pm 0.1 \mathrm{eV}$ two-photon eBE, which we attribute to ionisation from the $1 b_{1}$ molecular orbital of water. ${ }^{72}$ The residual of this fit is plotted as an inset and it can be fit with a Gaussian centred around $4.0 \pm$ $0.1 \mathrm{eV}$ (one-photon eBE) which we attribute to the photoelectron spectrum of the solvated electron. This value of eBE lies between the values of $4.5 \mathrm{eV}$ and $3.7 \mathrm{eV}$ 
obtained from careful measurements of the photoelectron spectra of solvated electrons at $5.8 \mathrm{eV}$ and $13.6 \mathrm{eV}$, respectively, ${ }^{2}$ and its observation is consistent with transient absorption measurements of solvated electrons being formed on a 200 fs timescale following $200 \mathrm{~nm}$ excitation. ${ }^{28}$ The residual on the low eBE edge of the solvated electron photoelectron spectrum can be attributed to $\mathrm{S}_{0}-\mathrm{D}_{0}$ ionisation (Fig. S4†).

Now we consider the effect of inelastic scattering on our photoelectron spectra. Recent careful measurements of UV photoelectron spectra of solvated electrons revealed that the measured eBE gradually increased with photon energy, indicating that the photoelectron energy diminished as a result of electron-solvent molecule inelastic scattering before emission from the surface of the liquid. ${ }^{1}$ Subsequent scattering simulations quantified the role of inelastic scattering on the photoelectron spectra. ${ }^{2}$ In order to investigate the impact of inelastic scattering on the peak positions and widths of our liquid-microjet photoelectron spectra, we plotted the eKEs of the maxima and full-width half-maxima (FWHM) of the Gaussians fitted to the $1^{1} \pi \pi^{*}-\mathrm{D}_{0} / \mathrm{D}_{1}$ processes, as a function of photon energy, alongside those for the $1^{1} \pi \pi^{*}-\mathrm{D}_{0}$ process for gas-phase phenol (Fig. 4). The peak widths do not seem to vary substantially with photon energy. The overall shapes of the three sets of data are very similar, with the peak eKEs remaining the same for both $275 \mathrm{~nm}$ and $265.5 \mathrm{~nm}$ spectra but then increasing approximately linearly. The gradient of the line fitted to the linearly increasing component of the plot for the gas-phase data is $1.02 \pm 0.02$, indicating that the propensity for

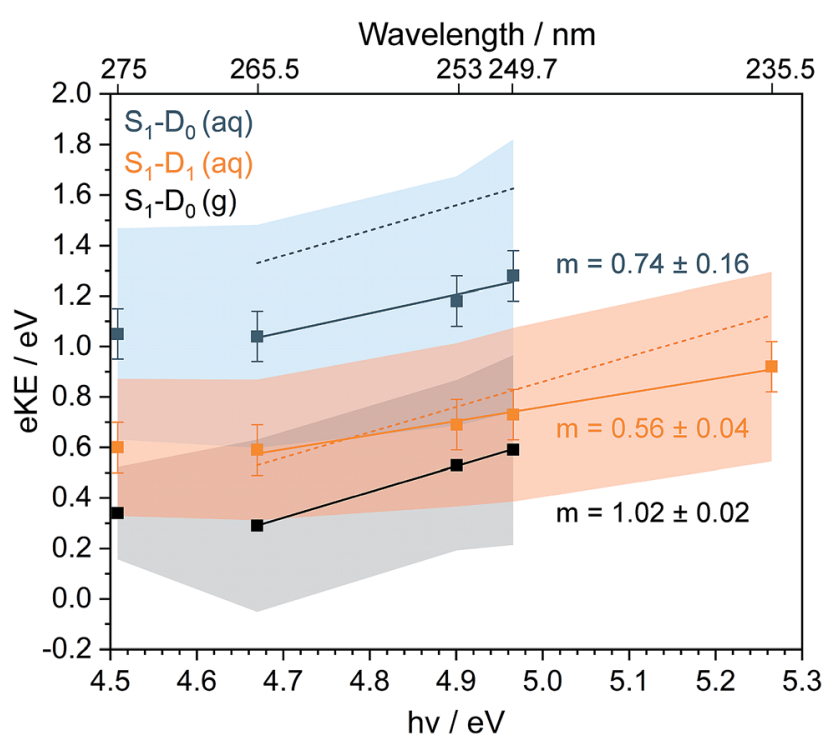

Fig. 4 Plots of fitted Gaussian peak maxima (data points) and full-width half maxima (shaded areas) corresponding to $S_{1}\left(1^{1} \pi \pi^{*}\right)-D_{0}$ in the gas phase $(g)$ and $S_{1}\left(1^{1} \pi \pi^{*}\right)-D_{0} / D_{1}$ in aqueous solution (aq), as a function of photon energy (bottom axis) and wavelength (top axis). Solid straight lines are fits to the higher photon energy data points, with gradients $m$ indicated. Dashed lines are peak maxima estimated using $S_{0}-D_{0} / D_{1}$ VIEs obtained from $X$ ray $\mathrm{PES}^{43}$ and the $\mathrm{S}_{0}-\mathrm{S}_{1}\left(1^{1} \pi \pi^{*}\right)$ AEE determined from the UV-vis absorption spectrum (Fig. 1, orange dashed line), assuming that vibrational energy is conserved during photoionisation. 
conserving vibrational energy during the $1^{1} \pi \pi^{*}-\mathrm{D}_{0}$ photoionisation process holds extremely well over this energy range. In contrast, the gradients of the lines fit to the peak positions corresponding to $1^{1} \pi \pi^{*}-\mathrm{D}_{0} / \mathrm{D}_{1}$ processes in aqueous phenol are less than unity. Moreover, these lines are shifted from the positions estimated using $S_{0}-D_{0} / D_{1}$ VIEs obtained from X-ray $P_{E S}{ }^{43}$ and the $S_{0}-1^{1} \pi \pi^{*}$ AEE determined from the UV-vis absorption spectrum (4.46 eV, Fig. 1), assuming that vibrational energy is conserved during photoionisation (dashed lines in Fig. 4). We used VIEs from X-ray PES measurements rather than our own measurement at $235.5 \mathrm{~nm}$ because we have not deconvoluted inelastic scattering from our $235.5 \mathrm{~nm}$ spectrum. Although it is possible that the propensity for conserving vibrational energy does not hold for aqueous phenol, we believe this is unlikely because it holds so well for gas-phase phenol, the UV-vis spectra of gas-phase and aqueous phenol are remarkably similar (Fig. 1) and the overall trends of the lines plotted in Fig. 4 are similar for aqueous phenol and gas-phase phenol. Thus, we believe the differences between the estimated peak positions and the actual peak positions can be attributed to inelastic electron scattering and note that our peak shifts are similar to those reported in ref. 2 over the same energy range.

It is this consideration of inelastic scattering that suggests the $235.5 \mathrm{~nm}$ photoelectron spectrum should be fit to three Gaussians rather than four. In the fit to four Gaussians (Fig. S5 $\dagger$ ), the $\mathrm{S}_{1}-\mathrm{D}_{0}$ peak is shifted to lower eBE than the $\mathrm{X}$ ray data whereas in the fit to three Gaussians (Fig. 3), it is shifted to higher eBE than the X-ray data. Although both fitted eBEs can be considered to be equivalent to the X-ray data within the experimental errors of both measurements, inelastic scattering would shift the measured peak to higher eBE, which suggests that the fit to three Gaussians is more appropriate. The wavelength dependence of inelastic scattering poses a particular problem for photoelectron spectra that span a wide range of eKEs, such as the $235.5 \mathrm{~nm}$ photoelectron spectrum. Although it is reasonable to fit Gaussians to a true photoelectron spectrum, Gaussians will be distorted by a wavelength-dependent inelastic scattering shift. Therefore, it is desirable to deconvolute inelastic scattering from a measured photoelectron spectrum to obtain a true photoelectron spectrum before fitting Gaussians. Unfortunately, this is not possible without detailed modelling of inelastic scattering across the relevant range of eKEs.

In Table 1 , we present our measured $S_{0}-D_{0} / D_{1}$ peak maxima together with our calculated VIEs and experimental and calculated values from the literature. For gas-phase phenol, our B3LYP/aug-cc-pvdz and EOM-IP-CCSD/6-31+G* methods both give VIEs that are within $0.2 \mathrm{eV}$ of the experimental AIEs $^{36,41}$ and are as good as other calculated VIEs reported in the literature. ${ }^{31,33,43}$ This gives us confidence that these are appropriate methods to investigate for the solution phase calculations.

For aqueous phenol, Ghosh et al. employed a $[\mathrm{phenol}]_{\mathrm{QM}}\left[\left(\mathrm{H}_{2} \mathrm{O}\right)_{\text {bulk }}\right]_{\mathrm{EFP}}(\mathrm{EOM}-$ IP-CCSD $\left./ 6-31+\mathrm{G}^{*}\right)$ method to calculate VIEs which were in very good agreement with X-ray PES measurements. ${ }^{43}$ However, we chose to include water molecules that were hydrogen-bonded to phenol in the QM region as well. For our aqueous phenol calculations, the VIE averaged over the 300 configurations saved from the MD calculations, using the $\left[\mathrm{phenol} \cdot\left(\mathrm{H}_{2} \mathrm{O}\right)_{5}\right]_{\mathrm{QM}}\left[\left(\mathrm{H}_{2} \mathrm{O}\right)_{n \geq 250}\right]_{\mathrm{EFP}}$ (B3LYP/aug-ccpvdz) method, is almost $0.4 \mathrm{eV}$ higher than the value obtained from X-ray PES measurements. ${ }^{43}$ The average VIE for the ten probable configurations (Section 2.2) calculated using the same method is slightly higher. Interestingly, using the 
Table 1 Calculated VIEs and measured IEs (or peak maxima) from $S_{0}$ to $D_{0}$ and $D_{1}$ in eV

\begin{tabular}{ccc}
\hline $\mathrm{D}_{0}$ & $\mathrm{D}_{1}$
\end{tabular}

\section{Gas-phase}

B3LYP/aug-cc-pvdz

EOM-IP-CCSD/6-31+G*

Experimental AIE (ref. 41)

Experimental AIE (ref. 36)

EOM-IP-CCSD/aug-cc-pvdz (ref. 31)

EOM-IP-CCSD/cc-pvtz (ref. 43)

$\operatorname{CAS}(7,8) / 6-31+\mathrm{G}^{*}$ (ref. 33)

\section{Aqueous solution}

$\left[\mathrm{Phenol} \cdot\left(\mathrm{H}_{2} \mathrm{O}\right)_{5}\right]_{\mathrm{QM}}\left[\left(\mathrm{H}_{2} \mathrm{O}\right)_{n \geq 250}\right]_{\mathrm{EFP}}$

$\left[\text { Phenol } \cdot\left(\mathrm{H}_{2} \mathrm{O}\right)_{5}\right]_{\mathrm{QM}}\left[\left(\mathrm{H}_{2} \mathrm{O}\right)_{n \geq 250}\right]_{\mathrm{EFP}}$

$\left[\mathrm{Phenol} \cdot\left(\mathrm{H}_{2} \mathrm{O}\right)_{5}\right]_{\mathrm{QM}}\left[\left(\mathrm{H}_{2} \mathrm{O}\right)_{n \geq 250}\right]_{\mathrm{EFP}}$

[Phenol $\left.\cdot\left(\mathrm{H}_{2} \mathrm{O}\right)_{5}\right]_{\mathrm{QM}}\left[\left(\mathrm{H}_{2} \mathrm{O}\right)_{n \geq 250}\right]_{\text {EFP }}$

Fitted peak maxima (Fig. 3)

X-ray PES (ref. 43)

$[\text { phenol }]_{\mathrm{QM}}\left[\left(\mathrm{H}_{2} \mathrm{O}\right)_{\text {bulk }}\right]_{\mathrm{EFP}}$ (ref. 43)

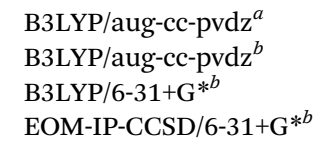

EOM-IP-CCSD/6-31+G*

$\begin{array}{ll}8.49 & \\ 8.34 & 9.22 \\ 8.51 & \\ 8.52 & 9.36 \\ 8.46 & 9.29 \\ 8.55 & \\ 7.99 & 8.67\end{array}$

8.17

$7.93(8.24)$

$7.94(8.25)$

$8.29(8.64) \quad 9.19(9.23)$

$8.0 \pm 0.1 \quad 8.9 \pm 0.1$

$7.8 \pm 0.1 \quad 8.6 \pm 0.1$

$7.9 \quad 8.6$

${ }^{a}$ Average of 300 unoptimised configurations from the MD sampling trajectory. ${ }^{b}$ Average of 10 configurations with $S_{0}$ energy close to the average $S_{0}$ energy (see text). Values in parentheses relate to configurations that have not been optimised using QM/EFP methods.

smaller $6-31+G^{*}$ basis set does not make much difference. The average VIE calculated using the EOM-IP-CCSD $/ 6-31+\mathrm{G}^{*}$ method is around $0.3 \mathrm{eV}$ higher than that calculated using the $\mathrm{B} 3 \mathrm{LYP} / 6-31+\mathrm{G}^{*}$.

We found that optimisation of the ten probable $\left[\text { phenol } \cdot\left(\mathrm{H}_{2} \mathrm{O}\right)_{5}\right]_{\mathrm{QM}}\left[\left(\mathrm{H}_{2} \mathrm{O}\right)_{n \geq 250}\right]_{\mathrm{EFP}}$ configurations lowered the calculated VIE values by around $0.1-0.3 \mathrm{eV}$. The average VIE calculated for the ten probable configurations following optimisation and using the B3LYP/aug-cc-pvdz method was $7.93 \mathrm{eV}$, which is in good agreement with the Xray PES measurement. Again, using the smaller $6-31+\mathrm{G}^{*}$ basis set made little difference. The average VIEs calculated for the optimised ten probable configurations using the EOM-IP-CCSD/6-31+G* method are around $0.4 \mathrm{eV}$ higher than the experimental values, although the difference between the $S_{0}-D_{0}$ and $S_{0}-D_{1}$ values $(0.9 \mathrm{eV})$ is reasonably close to the difference measured using X-ray PES. These calculations suggest that optimisation of configurations obtained from MD simulations could be important for determining accurate VIEs and that the simple DFT approach seems to work particularly well. It will be interesting to test this procedure for calculating VIEs on other molecules in aqueous solution to see if it is general rather than specific to phenol. Curiously, our EOM-IP-CCSD/EFP method, which includes water molecules that are hydrogen-bonded to phenol in the QM region, does not agree as well with the experimental measurements as the EOM-IP-CCSD/EFP method employed by Ghosh et al., which only included phenol in the QM region. ${ }^{43}$ However, it is worth noting that Ghosh et al. applied a correction to account for the effect of increasing basis set from 6-31+G(d) to cc-pVTZ.

Fig. 5 shows a simulated $\mathrm{S}_{0}-\mathrm{D}_{0}$ photoelectron spectrum obtained by plotting the distribution of VIEs calculated using the $\left[\text { phenol } \cdot\left(\mathrm{H}_{2} \mathrm{O}\right)_{5}\right]_{\mathrm{QM}}\left[\left(\mathrm{H}_{2} \mathrm{O}\right)_{n \geq 250}\right]_{\text {EFP }}$ (B3LYP/aug-cc-pvdz) method as a histogram with bin size $0.12 \mathrm{eV}$. The full-width half-maximum (FWHM) of the Gaussian fitted to the histogram is $0.86 \mathrm{eV}$. The 


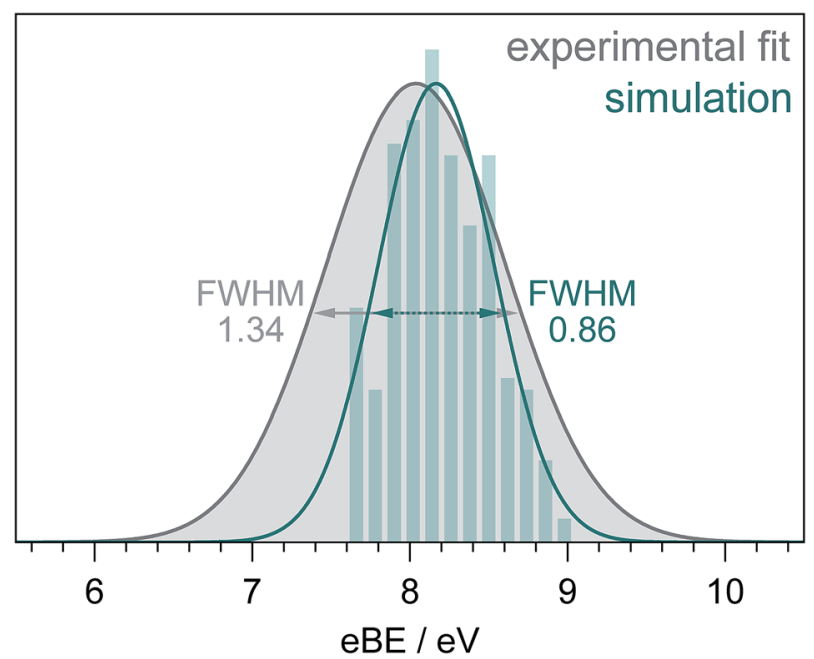

Fig. $5 S_{0}-D_{0}$ photoelectron spectra obtained from the fit to the $235.5 \mathrm{~nm}$ experimental data (Fig. 3) and simulation (see text). The full-width-half-maxima are shown for both Gaussian fits.

FWHM of the $\mathrm{S}_{0}-\mathrm{D}_{0}$ photoelectron spectrum obtained from the fit to the $235.5 \mathrm{~nm}$ spectrum (Fig. 3) is significantly larger (1.34 eV, similar to the X-ray PES FWHM ${ }^{43}$ ). The difference could be attributed to solute and solvent reorganisation, which is not accounted for in the simulation. The eKE dependence of inelastic scattering can also affect the widths of measured photoelectron spectra but to account for this properly requires detailed modelling of the electron scattering process. ${ }^{2}$

\section{Conclusion}

We have reported new MPI PES measurements of phenol in aqueous solution recorded using our recirculating liquid-microjet apparatus. Following resonant MPI via the $1^{1} \pi \pi^{*}$ state, the improved quality of these photoelectron spectra of phenol compared to those reported previously has allowed us to identify $1^{1} \pi \pi^{*}$ $\mathrm{D}_{0}$ and $1^{1} \pi \pi^{*}-\mathrm{D}_{1}$ ionisation processes and competing direct $\mathrm{S}_{0}-\mathrm{D}_{0}$ ionisation. Following resonant MPI via the $2^{1} \pi \pi^{*}$ state, we have observed the signature of solvated electrons. Following resonant MPI via the $1^{1} \pi \sigma^{*}$ state, we observed $1^{1} \pi \pi^{*}-\mathrm{D}_{0} / \mathrm{D}_{1}$ and $\mathrm{S}_{0}-\mathrm{D}_{0} / \mathrm{D}_{1}$ processes and, although we no longer find evidence for the formation of solvated electrons, we cannot rule out the possibility that solvated electrons are formed. Time-resolved PES measurements will be able to identify whether or not solvated electrons are formed following photoexcitation of the $1^{1} \pi \sigma^{*}$ state and such measurements are planned in our laboratory. The VIEs of photoexcited states of biologically relevant molecules in aqueous solution underpin ionisation and charge transfer processes and are thus important in radiation chemistry and biology. Solvated electrons, or more precisely presolvated electrons, are also known to play a role in inducing damage to DNA in aqueous solution.

By comparing the MPI photoelectron spectra of aqueous phenol and gas-phase phenol, we found that inelastic scattering resulted in peak shifts similar to those 
reported for photoelectron spectra of the solvated electron. ${ }^{1,2}$ The wavelength dependence of inelastic scattering poses a particular problem for interpreting broad photoelectron spectra and highlights a need for a robust way of deconvoluting the effect of inelastic scattering from liquid-phase photoelectron spectra. Quantifying the inelastic scattering of low energy electrons in aqueous solution is also crucially important for improving our understanding of the role of (pre-) solvated electrons in inducing damage in DNA in aqueous solution.

We have developed a QM/EFP protocol for calculating the VIEs of aqueous phenol. We found that DFT with a reasonably large basis set performed well. We also found that optimising the configurations obtained from MD simulations improved the value for the VIE. It will be interesting to investigate whether optimising greater numbers of configurations improves the agreement with experiment and to test the protocol on other molecules. The FWHM of our simulated $\mathrm{S}_{0}-\mathrm{D}_{0}$ photoelectron spectrum was less than that obtained from our fit to experimental data, which we attribute to solute and solvent reorganisation. Calculating solute and solvent reorganisation energies during ionisation is challenging, but we believe that high quality liquid-microjet photoelectron spectra together with analogous measurements in the gas-phase provide ideal benchmarks for theory.

\section{Conflicts of interest}

There are no conflicts to declare.

\section{Acknowledgements}

This work was supported by the EPSRC (EP/L005646/1), the Diamond Light Source (STU0157) and the Royal Society and Leverhulme Trust (SRF/R1/180079). We are grateful to Dr Anastasia Bochenkova (Moscow State University) for advice on the computational chemistry methods, to Professors Graham Worth (UCL) and Michael Robb (Imperial) for useful discussions and Dr Frank Otto for computational support.

\section{Notes and references}

1 Y. I. Yamamoto, S. Karashima, S. Adachi and T. Suzuki, J. Phys. Chem. A, 2016, 120, 1153-1159.

2 D. Luckhaus, Y.-i. Yamamoto, T. Suzuki and R. Signorell, Sci. Adv., 2017, 3, e1603224.

3 B. A. Barry, Photochem. Photobiol., 1993, 57, 179-188.

4 A. Acharya, A. M. Bogdanov, B. L. Grigorenko, K. B. Bravaya, A. V. Nemukhin, K. A. Lukyanov and A. I. Krylov, Chem. Rev., 2017, 117, 758-795.

5 A. Henley and H. Fielding, Int. Rev. Phys. Chem., 2019, 38, 1-34.

6 A. L. Sobolewski, W. Domcke, C. Dedonder-Lardeux and C. Jouvet, Phys. Chem. Chem. Phys., 2002, 4, 1093-1100.

7 M. N. R. Ashfold, G. A. King, D. Murdock, M. G. D. Nix, T. A. A. Oliver and A. G. Sage, Phys. Chem. Chem. Phys., 2010, 12, 1218-1238.

8 A. L. Sobolewski and W. Domcke, J. Phys. Chem. A, 2001, 105, 9275-9283.

9 W. Domcke and A. L. Sobolewski, Science, 2003, 302, 1693-1694. 
10 K. Daigoku, S.-I. Ishiuchi, M. Sakai, M. Fujii and K. Hashimoto, J. Chem. Phys., 2003, 119, 5149-5158.

11 C. M. Tseng, Y. T. Lee and C. K. Ni, J. Chem. Phys., 2004, 121, 2459-2461.

12 Z. Lan, W. Domcke, V. Vallet, A. L. Sobolewski and S. Mahapatra, J. Chem. Phys., 2005, 122, 224315.

13 M. N. R. Ashfold, B. Cronin, A. L. Devine, R. N. Dixon and M. G. D. Nix, Science, 2006, 312, 1637-1640.

14 M. G. Nix, A. L. Devine, B. Cronin, R. N. Dixon and M. N. R. Ashfold, J. Chem. Phys., 2006, 125, 133318.

15 C.-M. Tseng, Y. T. Lee, M.-F. Lin, C.-K. Ni, S.-Y. Liu, Y.-P. Lee, Z. F. Xu and M. C. Lin, J. Phys. Chem. A, 2007, 111, 9463-9470.

16 M. N. R. Ashfold, A. L. Devine, R. N. Dixon, G. A. King, M. G. D. Nix and T. A. A. Oliver, Proc. Natl. Acad. Sci. U. S. A., 2008, 105, 12701-12706.

17 O. P. Vieuxmaire, Z. Lan, A. L. Sobolewski and W. Domcke, J. Chem. Phys., 2008, 129, 224307.

18 G. A. King, T. A. A. Oliver, M. G. D. Nix and M. N. R. Ashfold, J. Phys. Chem. A, 2009, 113, 7984-7993.

19 A. Iqbal, L. J. Pegg and V. G. Stavros, J. Phys. Chem. A, 2008, 112, 9531-9534.

20 A. Iqbal, M. S. Y. Cheung, M. G. D. Nix and V. G. Stavros, J. Phys. Chem. A, 2009, 113, 8157-8163.

21 R. N. Dixon, T. A. A. Oliver and M. N. R. Ashfold, J. Chem. Phys., 2011, 134, 194303.

22 G. M. Roberts, A. S. Chatterley, J. D. Young and V. G. Stavros, J. Phys. Chem. Lett., 2012, 3, 348-352.

23 R. A. Livingstone, J. O. F. Thompson, M. Iljina, R. J. Donaldson, B. J. Sussman, M. J. Paterson and D. Townsend, J. Chem. Phys., 2012, 137, 184304.

24 S. G. Ramesh and W. Domcke, Faraday Discuss., 2013, 163, 73-94.

25 X. Xu, K. R. Yang and D. G. Truhlar, J. Chem. Theory Comput., 2013, 9, 36123625.

26 X. Xu, J. Zheng, K. R. Yang and D. G. Truhlar, J. Am. Chem. Soc., 2014, 136, 16378-16386.

27 K. R. Yang, X. Xu, J. Zheng and D. G. Truhlar, Chem. Sci., 2014, 5, 4661-4680.

28 T. A. A. Oliver, Y. Zhang, A. Roy, M. N. R. Ashfold and S. E. Bradforth, J. Phys. Chem. Lett., 2015, 6, 4159-4164.

29 C. Xie, J. Ma, X. Zhu, D. R. Yarkony, D. Xie and H. Guo, J. Am. Chem. Soc., 2016, 138, 7828-7831.

30 W. Xie and W. Domcke, J. Chem. Phys., 2017, 147, 184114.

31 J. W. Riley, B. Wang, J. L. Woodhouse, M. Assmann, G. A. Worth and H. H. Fielding, J. Phys. Chem. Lett., 2018, 9, 678-682.

32 G. Kumar, A. Roy, R. S. McMullen, S. Kutagulla and S. E. Bradforth, Faraday Discuss., 2018, 212, 359-381.

33 M. P. Taylor and G. A. Worth, Chem. Phys., 2018, 515, 719-727.

34 I. Sandler, J. J. Nogueira and L. González, Phys. Chem. Chem. Phys., 2019, 21, 14261-14269.

35 S. J. Harris, D. Murdock, Y. Zhang, T. A. A. Oliver, M. P. Grubb, A. J. Orr-Ewing, G. M. Greetham, I. P. Clark, M. Towrie, S. E. Bradforth and M. N. R. Ashfold, Phys. Chem. Chem. Phys., 2013, 15, 6567-6582.

36 M. J. S. Dewar and S. D. Worley, J. Chem. Phys., 1969, 50, 654.

37 T. P. Debies and J. W. Rabalais, J. Chem. Soc., Faraday Trans., 1972, 1, 355-370. 
38 J. P. Maier and D. W. Turner, J. Chem. Soc., Faraday Trans., 1973, 2, 521-531.

39 T. Kobayashi and S. Nagakura, Bull. Chem. Soc. Jpn., 1974, 47, 2563-2572.

40 M. H. Palmer, W. Moyes, M. Speirs and J. N. A. Ridyard, J. Mol. Struct., 1979, 52, 293-307.

41 R. J. Lipert and S. D. Colson, J. Chem. Phys., 1990, 92, 3240-3241.

42 S. L. Anderson, L. Goodman, K. Krogh-Jespersen, A. G. Ozkabak, R. N. Zare and C.-f. Zheng, J. Chem. Phys., 1985, 82, 5329-5339.

43 D. Ghosh, A. Roy, R. Seidel, B. Winter, S. Bradforth and A. I. Krylov, J. Phys. Chem. B, 2012, 116, 7269-7280.

44 A. Roy, R. Seidel, G. Kumar and S. E. Bradforth, J. Phys. Chem. B, 2018, 122, 3723-3733.

45 A. I. Krylov, Annu. Rev. Phys. Chem., 2008, 59, 433-462.

46 J. W. Riley, B. Wang, M. A. Parkes and H. H. Fielding, Rev. Sci. Instrum., 2019, 90, 083104.

47 G. K. Jarvis, M. Evans, C. Y. Ng and K. Mitsuke, J. Chem. Phys., 1999, 111, 3058. 48 A. D. Becke, J. Chem. Phys., 1993, 98, 5648-5652.

49 S. H. Vosko, L. Wilk and M. Nusair, Can. J. Phys., 1980, 58, 1200-1211.

50 C. Lee, W. Yang and R. G. Parr, Phys. Rev. B: Condens. Matter Mater. Phys., 1988, 37, 785-789.

51 P. J. Stephens, F. J. Devlin, C. F. Chabalowski and M. J. Frisch, J. Phys. Chem., 1994, 98, 11623-11627.

52 T. Clark, J. Chandrasekhar, G. W. Spitznagel and P. V. R. Schleyer, J. Comput. Chem., 1983, 4, 294-301.

53 M. J. Frisch, J. A. Pople and J. S. Binkley, J. Chem. Phys., 1984, 80, 3265-3269.

54 R. Krishnan, J. S. Binkley, R. Seeger and J. A. Pople, J. Chem. Phys., 1980, 72, 650-654.

55 J. Schirmer, Phys. Rev. A: At., Mol., Opt. Phys., 1982, 26, 2395-2416.

56 A. B. Trofimov and J. Schirmer, J. Phys. B: At., Mol. Opt. Phys., 1995, 28, 22992324.

57 Y. Shao, Z. Gan, E. Epifanovsky, A. T. Gilbert, M. Wormit, J. Kussmann, A. W. Lange, A. Behn, J. Deng, X. Feng, D. Ghosh, M. Goldey, P. R. Horn, L. D. Jacobson, I. Kaliman, R. Z. Khaliullin, T. Kuś, A. Landau, J. Liu, E. I. Proynov, Y. M. Rhee, R. M. Richard, M. A. Rohrdanz, R. P. Steele, E. J. Sundstrom, H. L. Woodcock, P. M. Zimmerman, D. Zuev, B. Albrecht, E. Alguire, B. Austin, G. J. O. Beran, Y. A. Bernard, E. Berquist, K. Brandhorst, K. B. Bravaya, S. T. Brown, D. Casanova, C.-M. Chang, Y. Chen, S. H. Chien, K. D. Closser, D. L. Crittenden, M. Diedenhofen, R. A. DiStasio, H. Do, A. D. Dutoi, R. G. Edgar, S. Fatehi, L. Fusti-Molnar, A. Ghysels, A. Golubeva-Zadorozhnaya, J. Gomes, M. W. Hanson-Heine, P. H. Harbach, A. W. Hauser, E. G. Hohenstein, Z. C. Holden, T.-C. Jagau, H. Ji, B. Kaduk, K. Khistyaev, J. Kim, J. Kim, R. A. King, P. Klunzinger, D. Kosenkov, T. Kowalczyk, C. M. Krauter, K. U. Lao, A. D. Laurent, K. V. Lawler, S. V. Levchenko, C. Y. Lin, F. Liu, E. Livshits, R. C. Lochan, A. Luenser, P. Manohar, S. F. Manzer, S.-P. Mao, N. Mardirossian, A. V. Marenich, S. A. Maurer, N. J. Mayhall, E. Neuscamman, C. M. Oana, R. Olivares-Amaya, D. P. O'Neill, J. A. Parkhill, T. M. Perrine, R. Peverati, A. Prociuk, D. R. Rehn, E. Rosta, N. J. Russ, S. M. Sharada, S. Sharma, D. W. Small, A. Sodt, T. Stein, D. Stück, Y.-C. Su, A. J. Thom, T. Tsuchimochi, V. Vanovschi, L. Vogt, O. Vydrov, T. Wang, M. A. Watson, 
J. Wenzel, A. White, C. F. Williams, J. Yang, S. Yeganeh, S. R. Yost, Z.-Q. You, I. Y. Zhang, X. Zhang, Y. Zhao, B. R. Brooks, G. K. Chan, D. M. Chipman, C. J. Cramer, W. A. Goddard, M. S. Gordon, W. J. Hehre, A. Klamt, H. F. Schaefer, M. W. Schmidt, C. D. Sherrill, D. G. Truhlar, A. Warshel, X. Xu, A. Aspuru-Guzik, R. Baer, A. T. Bell, N. A. Besley, J.-D. Chai, A. Dreuw, B. D. Dunietz, T. R. Furlani, S. R. Gwaltney, C.-P. Hsu, Y. Jung, J. Kong, D. S. Lambrecht, W. Liang, C. Ochsenfeld, V. A. Rassolov, L. V. Slipchenko, J. E. Subotnik, T. Van Voorhis, J. M. Herbert, A. I. Krylov, P. M. Gill and M. Head-Gordon, Mol. Phys., 2015, 113, 184-215.

58 M. J. Frisch, G. W. Trucks, H. B. Schlegel, G. E. Scuseria, M. A. Robb, J. R. Cheeseman, G. Scalmani, V. Barone, B. Mennucci, G. A. Petersson, H. Nakatsuji, M. Caricato, X. Li, H. P. Hratchian, A. F. Izmaylov, J. Bloino, G. Zheng, J. L. Sonnenberg, M. Hada, M. Ehara, K. Toyota, R. Fukuda, J. Hasegawa, M. Ishida, T. Nakajima, Y. Honda, O. Kitao, H. Nakai, T. Vreven, J. A. Montgomery Jr, J. E. Peralta, F. Ogliaro, M. Bearpark, J. J. Heyd, E. Brothers, K. N. Kudin, V. N. Staroverov, R. Kobayashi, J. Normand, K. Raghavachari, A. Rendell, J. C. Burant, S. S. Iyengar, J. Tomasi, M. Cossi, N. Rega, J. M. Millam, M. Klene, J. E. Knox, J. B. CrossV. Bakken, C. Adamo, J. Jaramillo, R. Gomperts, R. E. Stratmann, O. Yazyev, A. J. Austin, R. Cammi, C. Pomelli, J. W. Ochterski, R. L. Martin, K. Morokuma, V. G. Zakrzewski, G. A. Voth, P. Salvador, J. J. Dannenberg, S. Dapprich, A. D. Daniels, Ö. Farkas, J. B. Foresman, J. V. Ortiz, J. Cioslowski and D. J. Fox, Gaussian 09 Revision D.01, Gaussian Inc., Wallingford CT, 2013.

59 J. C. Phillips, R. Braun, W. Wang, J. Gumbart, E. Tajkhorshid, E. Villa, C. Chipot, R. D. Skeel, L. Kalé and K. Schulten, J. Comput. Chem., 2005, 26, 1781-1802.

60 R. B. Best, X. Zhu, J. Shim, P. E. M. Lopes, J. Mittal, M. Feig and A. D. Mackerell, J. Chem. Theory Comput., 2012, 3257-3273.

61 A. D. Mackerell, M. Feig and C. L. Brooks, J. Am. Chem. Soc., 2004, 126, 698699.

62 A. D. Mackerell, D. Bashford, M. Bellott, R. L. Dunbrack, J. D. Evanseck, M. J. Field, S. Fischer, J. Gao, H. Guo, S. Ha, D. Joseph-Mccarthy, L. Kuchnir, K. Kuczera, F. T. K. Lau, C. Mattos, S. Michnick, T. Ngo, D. T. Nguyen, B. Prodhom, W. E. Reiher, B. Roux, M. Schlenkrich, J. C. Smith, R. Stote, J. Straub, M. Watanabe, J. Wiórkiewicz-Kuczera, D. Yin and M. Karplus, J. Phys. Chem. B, 1998, 3586-3616.

63 M. S. Gordon, D. G. Fedorov, S. R. Pruitt and L. V. Slipchenko, Chem. Rev., 2012, 112, 632-672.

64 S. Bose, S. Chakrabarty and D. Ghosh, J. Phys. Chem. B, 2016, 120, 44104420.

65 D. Ghosh, D. Kosenkov, V. Vanovschi, J. Flick, I. Kaliman, Y. Shao, A. T. Gilbert, A. I. Krylov and L. V. Slipchenko, J. Comput. Chem., 2013, 34, 1060-1070.

66 P. N. Day, J. H. Jensen, M. S. Gordon, S. P. Webb, W. J. Stevens, M. Krauss, D. Garmer, H. Basch and D. Cohen, J. Chem. Phys., 1996, 105, 1968-1986.

67 J. P. Perdew, K. Burke and M. Ernzerhof, Phys. Rev. Lett., 1996, 77, 3865-3868. 68 J. P. Perdew, K. Burke and M. Ernzerhof, Phys. Rev. Lett., 1997, 78, 1396.

69 C. Adamo and V. Barone, J. Chem. Phys., 1999, 110, 6158-6170. 
70 A. A. Granovsky, Firefly version 8.2.0, http://classic.chem.msu.su/gran/firefly/ index.html.

71 M. W. Schmidt, K. K. Baldridge, J. A. Boatz, S. T. Elbert, M. S. Gordon, J. H. Jensen, S. Koseki, N. Matsunaga, K. A. Nguyen, S. Su, T. L. Windus, M. Dupuis and J. A. Montgomery Jr, J. Comput. Chem., 1993, 14, 1347-1363.

72 B. Winter and M. Faubel, Chem. Rev., 2006, 106, 1176-1211. 